\title{
Remote Monitoring and Control for an Isolate Prototype Substation Model
}

\author{
Sandhya Rani $\mathrm{G}^{1, *}$, Vijaya Laxmi $\mathrm{U}^{1}$, Srividya Devi $\mathrm{P}^{2}$, Naga Sandhya Rani $\mathrm{M}^{1}$ \\ ${ }^{1}$ Assitant Professor, EEE Department, GRIET, Hyderabad \\ ${ }^{2}$ Associate Professor, EEE Department, GRIET, Hyderabad
}

\begin{abstract}
The objective of this paper is to monitor the electrical parameters like voltage, current, etc., remotely and display all the obtained real time values for a substation isolate. This paper is furnished to assure the load and electrical system equipment by the activation of relay, whenever the acquired parameters exceed the predefined values. Generally, this Proposed system design makes use of microcontroller, but the prototype of this circuit modelled in Proteus and can be executed by using ATmega 168 microcontroller. When supply is given to the designed hardware, all the sensors start sensing their respective parameters i.e., voltage, current, temperature etc., and modernize all the values on the display. Comparison between the problem-solving time values and the preordained values is continuously carried out by the microcontroller, if any of these values go beyond the pre-defined values, it sends fault alert to the relay, updates it on the screen and sends the same as an SMS through GSM for the rectification.
\end{abstract}

\section{Introduction}

Electricity is an exceedingly important and effective mode of energy. It plays a crucial act in contemporary world. Power systems are of highly complex and nonlinear networks. Electric energy transmission and distribution structures are more required to function correctly and reliably to guarantee each continuity and high-satisfactory of supply [1]. Electrical power system is one of the key elements which play a vital role in both domestic and international framework and any damage to this system may lead to both fundamental direct and oblique impact at the countrywide financial system and security. As the functioning of power systems is hugely time-critical, low-latency communication should be considered for most control and monitoring applications [2]. Many advanced technologies are being developed today, even though electricity still suffers from power abnormalities and blackouts. Hence it has become necessary for a utility company to shift to automated analysis of the complex distribution network. The increasing complexity of huge electric power systems has resulted in a more prominent requirement for maintenance in order to keep a reliable supply of power [3].

The security and control systems comprise all segments and frameworks used to screen, control, ensure, and robotize the substation [4]. Home automation makes home more customized, comfortable, efficient, and riskless. Numerous applications of machine learning techniques to control power system security have been accounted [5]. These days research acquaints machine learning techniques to distinguish the flaw type, area identification and prediction [6]. Machine learning is appreciable for Problems for which the present solution depends up on a lot of fine adjustment or long details of rules: one Machine Learning design can often clarify code and perform better than the traditional approach. The applications of Machine Learning are 1) Analyzing images of products 2) Detecting tumors in brain scans 3) Automatically classify news articles. The harmony among supply and demand is the foundation of power system operations [7]. The purpose of this is to protect the load and substation equipment from damage due to any kind of faults like overload, short circuit, high temperature etc., Meanwhile, during fault an alert is sent by an SMS through GSM module and the same is displayed on the LCD display. Hence, by this it becomes very easy to notice the fault occurrence in the substation also the type of fault.

\footnotetext{
*Corresponding author:sandhyaranister@gmail.com
} 


\section{System Design and Modelling}

The aim of this paper is to sense and monitor the real time electrical parameters like voltage, current, frequency and temperature continuously. And if any of these values of parameters like current, frequency exceed the pre-defined or pre-set values, relay is triggered to isolate the load from the supply.

Objectives

$>$ Power delivered should be made reliable and compatible by speedy detection and isolation of fault.

$>$ Quality of service should be impressed by ensuring real time monitoring.

$>$ Ensure the sensing of electrical parameters remotely.

$>$ Keep continuous track of real time values meanwhile maintain power supply continuity.

$>$ Should be more economical and reduce the manual labor cost.

The Block diagram which is shown in Fig. 1 represents a step-down transformer is used. It is because conversion of a.c to d.c is easy for lower voltages and moreover all the electronic components in the setup work on the lower d.c voltage ranges. Along with the micro controller or Regulated power supply board consists of a diode Bridge Rectifier unit made up of 4, 1N4007 rectifier diodes, a Capacitor of $470 \mu \mathrm{F}$, a IC7805 Voltage-regulator and 2, $0.1 \mu \mathrm{F}$ Film-capacitors. The stepped down 12 Volt, $50 \mathrm{~Hz}$ ac is given to the bridge-rectifier that converts it to DC voltage of $12 \mathrm{~V}$ which is pulsating in nature, to smoothen the pulses it is passed through the filter i.e., a capacitor of $470 \mu \mathrm{F}$. Along with the micro controller or Arduino uno many components need $5 \mathrm{~V}$ as $+\mathrm{Vcc}$, so a voltage regulator IC7812 is used to convert $12 \mathrm{~V}$ dc to $5 \mathrm{~V}$ constant DC. Arduino microcontroller is used as easy to program [8].

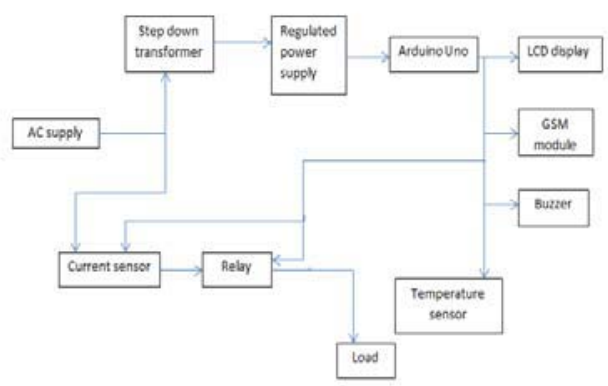

Fig. 1.Block Diagram of the System design for Substation Control system

The recent development in power electronic equipment's made the converters to required part of the industrial implementation. Particularly the AC-DC converters became leading because of the growing DC power needs in high power applications [9]. This type of converters behaves as nonlinear loads due to the utilise of solidstate switches and draw non sinusoidal currents from the supply mains [10]. A full wave bridge rectifier chip is readily available in the market, or it can be easily constructed using four diodes.

Operation of the diode bridge rectifier is explained as:

$>$ Right side upper diode and left side lower diodes get forward biased and remaining two are reverse biased during positive half cycle.

$>$ Left side upper diode and right-side lower diodes get forward biased and remaining two are reverse biased during negative half cycle.

Whichever diodes may be forward biased, or reverse biased the direction of output doesn't change for each half cycle. This is how a full wave bridge rectifier converts ac quantity to dc quantity. These rectifiers are part of many industrial applications [11]. These rectifiers experience the ill effects of issues, for example poor power quality in terms of current harmonics, voltage distortions, poor power factor, low efficiency at input ac mains. Different filters are utilized at input side and output side to lessen the ripple content in DC output, to boost efficiency, to decrease harmonics in Line current [12].

Generally, the rectified dc output has ripples in the waveform. This is due to the presence of ac component in the rectified dc. Hence to smoothen these ripples, rectified dc output is sent to a filter circuit that is an electrolytic capacitor. In general, shunt capacitor filter is widely used for smoothening out the ripples in $\mathrm{dc}$ voltage. It is a system or a device is shown in Fig. 4 is an integrated circuit designed to regulate the output voltage to a constant value. Whenever a stable voltage output is required to provide supply for some electronic device's voltage regulator is preferred. In this proposed circuit IC $7805[13]$ is used. The first two digits 78XX represent the family of regulators and the last two digits XX05 represent the value of voltage it maintains across its output terminal shown in Fig. 2

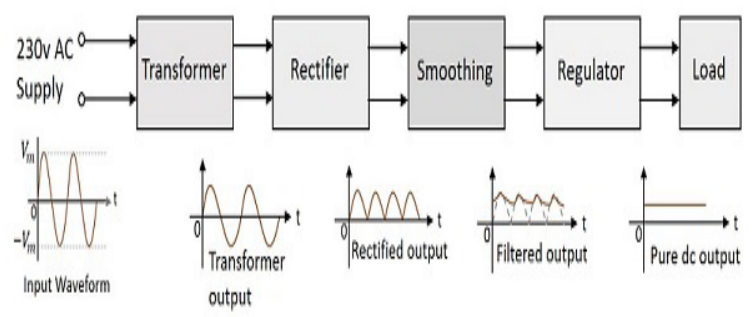

Fig. 2. Regulated Power Supply Block Diagram.

\section{Proposed Methodology}

As Modern power systems become bigger and more muddled, it is very predominant to enhance the credibility and security of their electric power supply [14].The main components used in this project are (230/12) V Transformer, regulated power supply board, Arduino UNO [15], GSM module, Current sensor (ACS712), Temperature sensor (LM35), SPDT relay, 
LCD display(16x2), a Piezo buzzer and an incandescent bulb is used as load shown in Fig.3.The LM35 sensor is an analog sensor that converts temperature into electrical quantities in the form of voltage [16]. The Transformer used step downs the $230 \mathrm{~V}$ ac supply to $12 \mathrm{~V}$. This $12 \mathrm{~V}$ ac supply is passed to Regulated Power Supply Board which consists of rectifiers and regulators; firstly, the converted $12 \mathrm{~V}$ will be passed over bridge rectifier which readily converts the ac supply to dc. The converted voltage may not be exactly $12 \mathrm{~V}$, so it is sent to $12 \mathrm{~V}$ voltage regulator named IC7812. As the controller board requires $5 \mathrm{~V}$, the $12 \mathrm{~V}$ dc voltage is passed through other $5 \mathrm{~V}$ regulator named IC7805 so that exact $5 \mathrm{~V}$ supply is obtained.

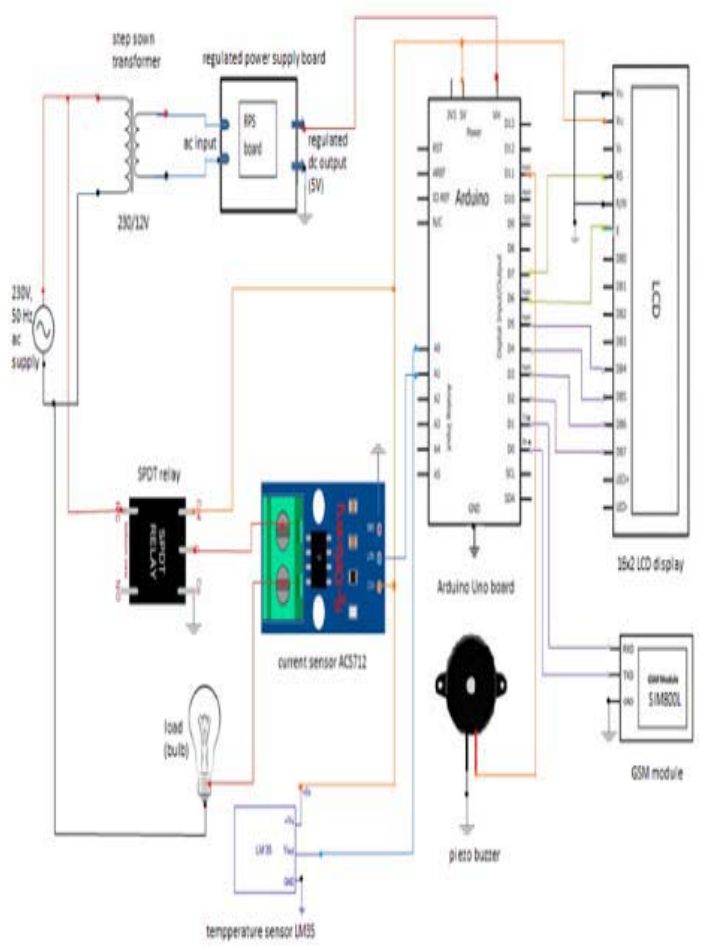

Fig. 3. Design of Proposed Circuit in Proteus software

LCD display, current sensor, temperature sensor, buzzer and relay are given power supply from Arduino board only. As GSM module works for $4.5 \mathrm{~V}$ unless $5 \mathrm{~V}$ so it takes $5 \mathrm{~V}$ de supply from RPS board and regulates it to $4.5 \mathrm{~V} \mathrm{dc}$ through the inbuilt regulators. Arduino UNO acts as core to the whole project. It is connected to 3 output and 2 input units that is current sensor and temperature sensor acts as input units whereas buzzer, SPDT relay, LCD display acts as output units. Arduino is the one which works as a processor to carry out the circuit operation in the project. An additional technology added to this project is GSM module which can send personalized messages to the person in charge over the substation. Current sensor and Temperature sensor send the sensed data to the controller continuously. Arduino is the one which takes decision on the output to be displayed. Relay trips the circuit when a signal is received from the Arduino and the buzzer gets alarmed too [17-18]. The Transformer used step downs the $230 \mathrm{~V}$ ac supply to $12 \mathrm{~V}$. This $12 \mathrm{~V}$ ac supply is passed to Regulated Power Supply Board which consists of rectifiers and regulators; firstly, the converted $12 \mathrm{~V}$ will be passed over bridge rectifier which readily converts the ac supply to dc. The converted voltage may not be exactly $12 \mathrm{~V}$, so it is sent to $12 \mathrm{~V}$ voltage regulator named IC7812. A stream diagram for the proposed displayed in Fig. 4 as the controller board requires $5 \mathrm{~V}$, the $12 \mathrm{~V}$ dc voltage is passed through other $5 \mathrm{~V}$ regulator named IC7805 so that exact $5 \mathrm{~V}$ supply is obtained.

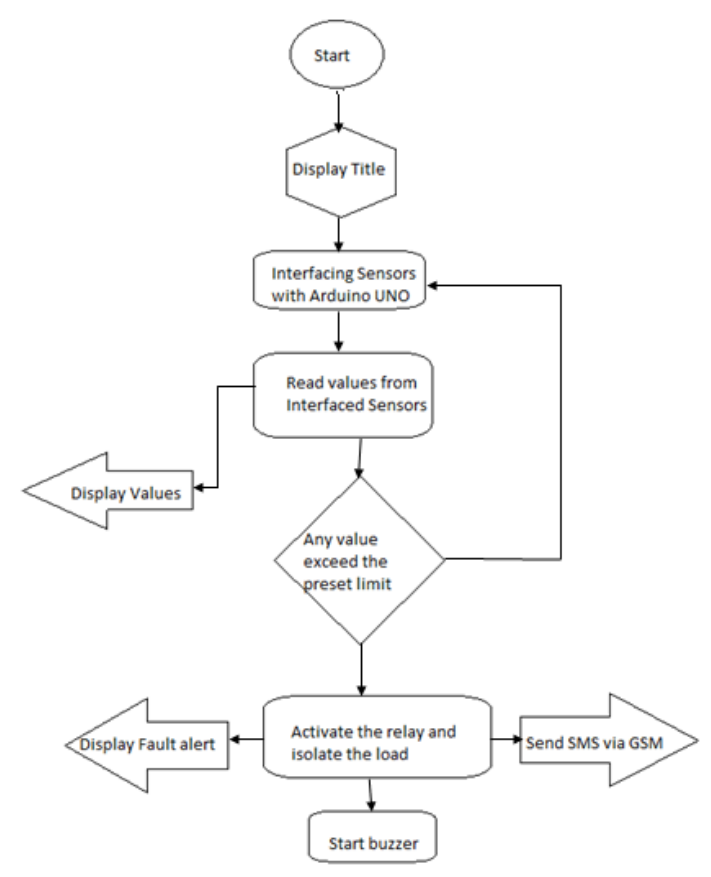

Fig. 4. Flow chart of the Prototype operation of Control

LCD display, current sensor, temperature sensor, buzzer and relay are given power supply from Arduino board only. As GSM module works for $4.5 \mathrm{~V}$ unless $5 \mathrm{~V}$ so it takes $5 \mathrm{~V}$ dc supply from RPS board and regulates it to $4.5 \mathrm{~V} \mathrm{dc}$ through the inbuilt regulators. When the supply is given to the circuit current sensor and temperature sensor start sensing the respective parameters and continuously send the data i.e. instantaneous current and temperature values to the controller i.e. Arduino. The controller reads the data and displays the current and temperature values on the LCD display. Voltage and frequency values derived from the sensed current and temperature values are also displayed on the screen. The controller is also programmed to check the current and temperatures values with the pre-set values. If any parameter values deviate from the pre-set values, the controller sends signal to the relay to isolate the load from the supply and buzzer to produce an alarm [19-20]. Along with that a text message is sent to the person in charge via GSM module and the same message is displayed on the LCD screen. 
When over current is detected, then it sends a message as "Over Current Detected". If high temperature is detected, then it sends the message as "High Temperature Detected". Hence monitoring of the substation is achieved along with protecting the load and substation equipment from damage due to power abnormalities.

\section{Experimental Results}

In Fig. 5 and Fig. 6 the Hardware of the proposed circuit is shown. As explained in the working principle, the proposed circuit monitors the instantaneous electrical parameters like voltage, current, temperature and frequency.

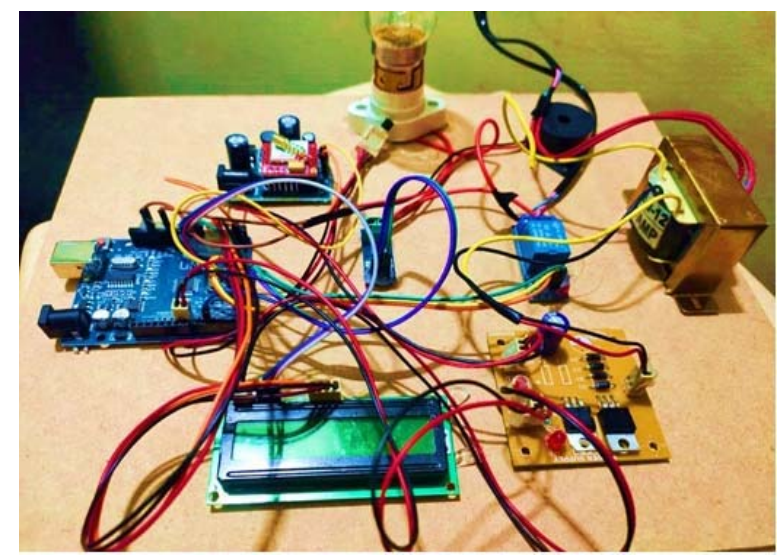

Fig. 5. Hardware Unit of Proposed Project for substation control

The controller reads the data from sensors and displays the current and temperature values on the LCD display. usually based on temperature for a material elevations can be seen[21] Voltage and frequency values derived from the sensed current and temperature values are also displayed on the screen as shown in Fig. 7.

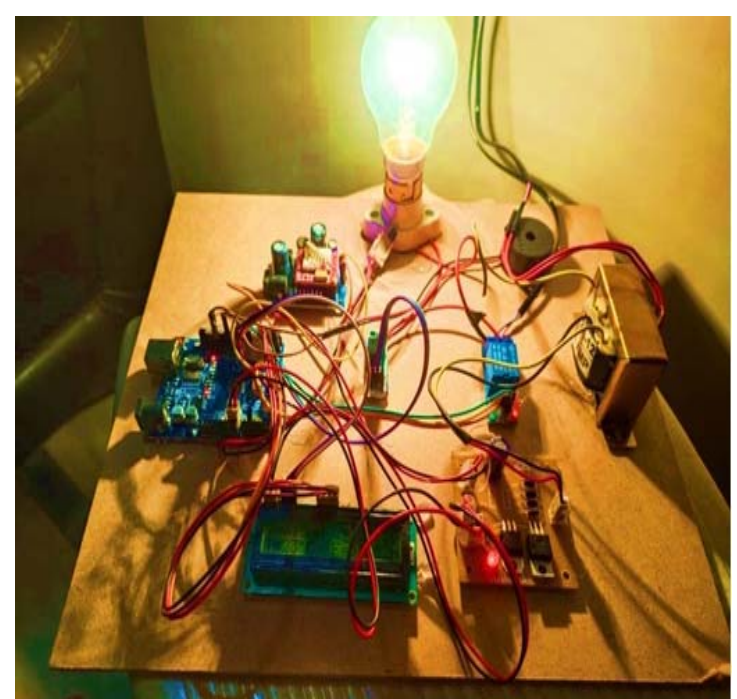

Fig. 6. When Power Supply is given to the Circuit

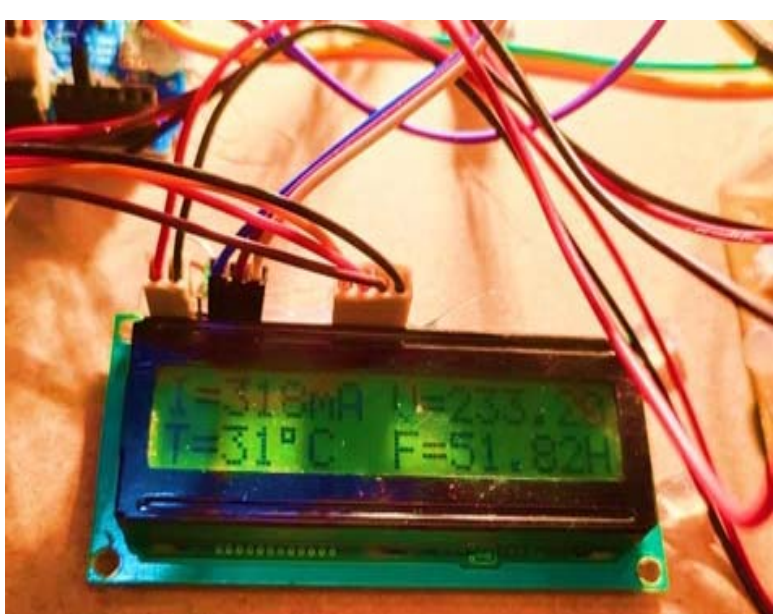

Fig.7.LCD Displaying Real-Time Values.

The controller is also programmed to check the current and temperatures values with the pre-set values. If current value deviates from the pre-set value, the controller sends signal to the relay to isolate the load from the supply and buzzer to produce an alarm. Along with that a text message "Over Current" is displayed on the LCD screen.

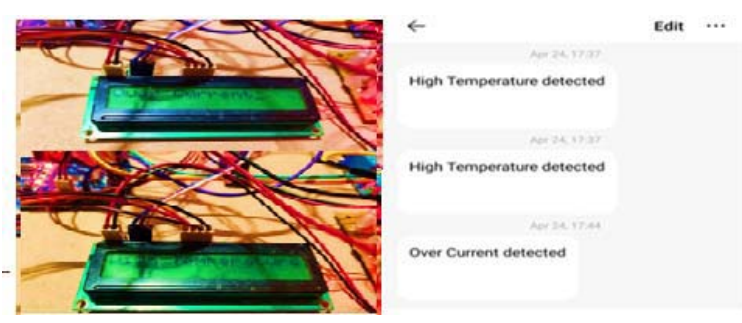

Fig. 8. Over Current/High Temperature Detection with an personal mobile message

If temperature value deviates from the pre-set value, the controller sends signal to the relay to isolate the load from the supply and buzzer to produce an alarm. Along with that a text message "High Temperature" is displayed on the LCD screen. A text message is sent to the person in charge of substation via GSM module along with the message displayed on the LCD screen as shown in Fig 8.

\section{Conclusion:}

This Paper on Substation monitoring and control system ensures the quality of power transferred in the substation can be improved. And, real time monitoring of electrical parameters is done, which helps in the safety measures of the substation and its equipment. Required parameters such as current, frequency, temperature and voltage can be monitored, and their status is also acquired remotely using GSM module. The data monitoring of the substation helps to identify the faults, trips and breakages occur in the Electric equipment, which gives the quick response to tackle any such event. The paper 
has been implemented and designed using latest technology. Each module was reasoned out and carefully implemented. Thus, this experimental setup was successfully designed and tested. The microcontroller can also be reprogrammed to also receive text messages to know the instantaneous value of any parameter. Storing the parameters values will provide better monitoring of the substation. It can be achieved by integrating this designed circuit with IoT helps in storing the parameter values in the cloud. Visual monitoring of the substation can be done by installing wireless cameras in the substation switchyard where the fault is more likely to happen.

\section{References}

1. A. Bogias, H. Griffiths, N. Harid S. Robson and A. Haddad, IEEE access, 14, (2016)

2. Long Zhao Igor Matsuo Yuhao Zhou Wei-Jen, IEEE Transc. On Industry Apls.,99 (2019)

3. W.L. Chan, T.M. Chan, S.L. Pang,A.T.P IEEE Transc. on Power Delivery, 12, (1997)

4. Jing $\mathrm{Ma}$, Jie $\mathrm{Wu}$, and Zengping Wang, International conf. on crtical Infrastructure, (2010)

5. Tilottama Goswami, Uponika Barman Roy, IEEE region 10 conf., (2019)

6. Song Tan, Wen-Zhan Song, Michael Stewart, Junjie Yang, Lang Tong, "IEEE Transactions on Smart Grid. 9, (2018)

7. Tarlochan Kaur, JaimalaGambhir, Sanjay Kumar, International conf. on power electronics, (2016)

8. P. SaravanaPrakash,R. Kalpana, Khimavath Sai Chethana and Bhim Singh, IEEE Transc. on Industry Apls, 57, (2021)

9. A. Siebert, A. Troedson and S. Ebner, 38, 934 (2002)

10. G.V. Aparna, G Suresh Babu,T. Murali Krishna , ICRIEECE, (2018)

11. Ahmed Al Mansur, Abdullah Al Bashit, A.S.M MahfuzurRahman,Md.ShahinurAlam, Hasina Begum, IJSER,4, (2013)

12. D. Florean, M. Pinelli, , P. Tomasin, International symposium on electromagnetic compatibility, (2000).

13. Sungmin Park, Eunjae Lee, Wonkun Yu, Heungjae Lee, Jeonghoon Shin, IEEE Transc. On Smart Grid, 4, 1 (2013).

14. PVS.DivyaDhatri,M.Pachiyannan,

K.JyothiSwaroopaRani, International Conf. on Signal Processing and Communication (2019)

15. TigorHamonangan Lukman AdlinHarahap, International Conf. on Electrical, Telecommunication and Computer Engineering, (2020)

16. Rani, V.U., Sridevi, J., Sai, P.M., IEEE- SeFet (2021)

17. Lakshmi Swarupa, M., Tulasi Ram Das, G., Sridevi, International Conf. on Renewable Energy Integration into Smart Grids: 127, (2020)

18. Sridevi, J., Rani, V.U., Rao, B.L. 2019 IEEE International Conf. on Electrical, Control and Instrumentation Engineering, (2019)

19. Sridevi, J., Amarnath, J., Govinda Rao, G., International Review on Modelling and Simulations , 4,2485 (2011)

20. Sridevi, J., Amarnath, J., Govinda Rao, G., International Review on Modelling and Simulations, 4,1799 (2011)
21. Singh, S.K., Gupta, A.K., Mahesh, K. CIRP Journal of Manufacturing Science and Technology, 3, 73(2010) 